\title{
On lengths on semisimple groups
}

\author{
Yves de Cornulier
}

May 21, 2009

\begin{abstract}
We prove that every length on a simple group over a locally compact field, is either bounded or proper.
\end{abstract}

\section{Introduction}

Let $G$ be a locally compact group. We call here a semigroup length on $G$ a function $L: G \rightarrow \mathbf{R}_{+}=[0, \infty[$ such that

- (Local boundedness) $L$ is bounded on compact subsets of $G$.

- (Subadditivity) $L(x y) \leq L(x)+L(y)$ for all $x, y$.

We call it a length if moreover it satisfies

- (Symmetricalness) $L(x)=L\left(x^{-1}\right)$ for all $x \in G$.

We do not require $L(1)=1$. Note also that local boundedness weakens the more usual assumption of continuity, but also include important examples like the word length with respect to a compact generating subset. See Section 2 for further discussion. Besides, a length is called proper if $L^{-1}([0, n])$ has compact closure for all $n<\infty$.

Definition 1.1. A locally compact group $G$ has Property $P L$ (respectively strong Property $P L$ ) if every length (resp. semigroup length) on $G$ is either bounded or proper.

We say that an action of a locally compact group $G$ on a metric space is locally bounded if $K x$ is bounded for every compact subset $K$ of $G$ and $x \in X$. This relaxes the assumption of being continuous. The action is bounded if the orbits are bounded. If $G$ is locally compact, the action is called metrically proper if for every bounded subset $B$ of $X$, the set $\{g \in G \mid B \cap g B \neq \emptyset\}$ has compact closure.

Proposition 1.2. Let $G$ be a locally compact group. Equivalences: 
(i) G has Property PL;

(ii) Any action of $G$ on a metric space, by isometries, is either bounded or metrically proper;

(ii') Any action of $G$ on a metric space, by uniformly Lipschitz transformations, is either bounded or metrically proper;

(iii) Any action of $G$ on a Banach space, by affine isometries, is either bounded or metrically proper.

When $G$ is compactly generated, Property PL can also be characterized in terms of its Cayley graphs.

Proposition 1.3. Let $G$ be a locally compact group. If $G$ has strong Property PL (resp. Property $P L$ ), then for any subset $S$ (resp. symmetric subset) generating $G$ as a semigroup, either $S$ is bounded or we have $G=S^{n}$ for some $n$. If moreover $G$ is compactly generated, then the converse also holds.

I do not know if the converse holds for general locally compact $\sigma$-compact groups. Also, I do not know any example of a locally compact group with Property PL but without the strong Property PL.

If a locally compact group is not $\sigma$-compact, then it has no proper length and therefore both Property PL and strong Property PL mean that every length is bounded. Such groups are called strongly bounded (or are said to satisfy the Bergman Property); discrete examples are the full permutation group of any infinite set, as observed by Bergman [Be] (see also [C]). However the study of Property $\mathrm{PL}$ is mainly interesting for $\sigma$-compact groups, as it is then easy to get a proper length (it is more involved to obtain a continuous proper length; this is done in [St], based on the Birkhoff-Kakutani metrization Theorem).

The main result of the paper is.

Theorem 1.4. Let $\mathbf{K}$ be a local field (that is, a non-discrete locally compact field) and $G$ a simple linear algebraic group over $\mathbf{K}$. Then $G_{\mathbf{K}}$ satisfies strong Property $P L$.

This result was obtained by Y. Shalom [Sh] in the case of continuous Hilbert lengths, i.e. lengths $L$ of the form $L(g)=\|g v-v\|$ for some continuous affine isometric action of $G$ on a Hilbert space, with an action of a group of K-rank one. Some specific actions on $L^{p}$-spaces were also considered in [CTV].

My original motivation was to extend Shalom's result to actions on $L^{p}$-spaces, but actually the result turned out to be much more general. However, even for isometric actions on general Banach spaces, we have to prove the result not only in $\mathbf{K}$-rank one, but also in higher rank, in which case the reduction to $\mathrm{SL}_{2}$ requires some careful arguments. 
The first step is the case of $\mathrm{SL}_{2}(\mathbf{K})$; it is elementary but it seems that it has not been observed so far (even for $\mathbf{K}=\mathbf{R}$ ).

Then with some further work, and making use of the Cartan decomposition, we get the general case. In the case of rank one, this second step is straightforward; this was enough in the case of Hilbert lengths considered in [Sh in view of Kazhdan's Property $T$ for simple groups of rank $\geq 2$ (which states that every Hilbert length is bounded), but not in general as there always exist unbounded lengths.

Remark 1.5. It is necessary to consider lengths bounded on compact subsets. Indeed, write $\mathbf{R}$ as the union of a properly increasing sequence of subfields $K_{n}$. (For instance, let $I$ be a transcendence basis of $\mathbf{R}$ over $\mathbf{Q}$, write $I$ as the union of a properly increasing sequence of subsets $I_{n}$, and define $K_{n}$ as the set of reals algebraic over $\mathbf{Q}\left(I_{n}\right)$.) If $G=G(\mathbf{R})$ is a connected semisimple group, then $\ell(g)=\min \left\{n \mid g \in G\left(K_{n}\right)\right\}$ is an unbounded symmetric (and ultrametric) nonlocally bounded length on $G$. However $\ell$ is not bounded on compact subsets and $\{\ell \leq n\}$ is dense provided $G$ is defined over $K_{n}$, and this holds for $n$ large enough.

Also, if $G=G(\mathbf{C})$ is complex and non-compact, if $\alpha$ is the automorphism of $G$ induced by some non-continuous field automorphism of $\mathbf{C}$, and if $\ell$ is the word length with respect to some compact generating set, then $\ell \circ \alpha$ is another example of a non-locally bounded length neither bounded nor proper.

Finally, it is convenient to have a result for general semisimple groups.

Proposition 1.6. Let $\mathbf{K}$ be a local field and $G$ a semisimple linear algebraic group over $\mathbf{K}$. Let $L$ be a semigroup length on $G(\mathbf{K})$. Then $L$ is proper if (and only if) the restriction of $L$ to every non-compact $\mathbf{K}$-simple factor $G_{i}(\mathbf{K})$ is unbounded.

This proposition relies on Theorem 1.4, from which we get that $L$ is proper on each factor $G_{i}(\mathbf{K})$, and an easy induction based on the following lemma, of independent interest.

Lemma 1.7. Let $H \times A$ be a locally compact group. Suppose that $H=G(\mathbf{K})$ for some $\mathbf{K}$-simple linear algebraic group over $\mathbf{K}$. Let $L$ be a semigroup length on $G$, and suppose that $L$ is proper on $H$ and $A$. Then $L$ is proper.

Here are some more examples of PL-groups, beyond semisimple groups.

Proposition 1.8. Let $K$ be a compact group, with a given continuous orthogonal representation on $\mathbf{R}^{n}$ for $n \geq 2$, so that the action on the 1-sphere is transitive (e.g. $K=\mathrm{SO}(n)$ or $K=\mathrm{SU}(m)$ with $2 m=n \geq 4$ ). Then the semidirect product $G=\mathbf{R}^{n} \rtimes K$ has strong Property $P L$.

Proposition 1.9. Let $\mathbf{K}$ be a non-Archimedean local field with local ring $\mathbf{A}$. Then the group $\mathbf{K} \rtimes \mathbf{A}^{*}$ has strong Property PL.

Note that the locally compact group $\mathbf{K} \rtimes \mathbf{A}^{*}$ is not compactly generated. 


\section{Discussion on lengths}

We observe here that our results actually hold for more general functions than lengths. Namely, call a weak length a function $G \rightarrow \mathbf{R}_{+}$which is locally bounded and satisfies

(Control Axiom) There exists a non-decreasing function $\phi: \mathbf{R}_{+} \rightarrow \mathbf{R}_{+}$such that for all $x, y$, we have $L(x y) \leq \phi(\max (L(x), L(y))$ for all $x, y$.

Note that every semigroup length satisfies the control axiom with $\phi(t)=2 t$. Besides, if $L, L^{\prime}$ are two weak lengths on $G$, say that $L$ is coarsely bounded by $L^{\prime}$ and write $L \preceq L^{\prime}$ if $L \leq u \circ L^{\prime}$ for some proper function $u: \mathbf{R}_{+} \rightarrow \mathbf{R}_{+}$, and that $L$ and $L^{\prime}$ are coarsely equivalent, denoted $L \simeq L^{\prime}$, if $L \preceq L^{\prime} \preceq L$. Here is a series of remarks concerning various definitions of lengths.

1. (Vanishing at 1) Let $L$ be a length (resp. weak length). Set $L^{\prime}(x)=L(x)$ if $x \neq 1$ and $L^{\prime}(1)=0$. We thus get another length (resp. weak length), and obviously $L$ and $L^{\prime}$ are coarsely equivalent.

2. (Continuity) A construction due to Kakutani allows to replace any length by a coarsely equivalent length which is moreover continuous (see $\mathrm{Hj}$, Theorem $7.2])$.

3. For every weak length $L$, there exists a semigroup length $L_{1}$ coarsely equivalent to $L$. The argument is as follows: we can suppose that $\phi$, the controlling function involved in the definition of weak length, is a bijection from $\mathbf{R}_{+}$to $[\phi(0),+\infty$ [ satisfying $\phi(t) \geq t+1$ for all $t$; then it makes sense to define inductively $\alpha(t)=t / \phi(0)$ for all $t \in[0, \phi(0)]$ and $\alpha(t)=\alpha\left(\phi^{-1}(t)\right)+1$ for $t \geq \phi(0)$. By construction, $\alpha(\phi(t)) \leq \alpha(t)+1$ for all $t$. Thus $L_{1}^{\prime}(x)=\alpha(L(x))$ satisfies the quasi-ultrametric axiom $L_{1}^{\prime}(x y) \leq \max \left(L_{1}^{\prime}(x), L_{1}^{\prime}(y)\right)+1$, and consequently $L_{1}=1+L_{1}^{\prime}$ is a semigroup length. Moreover, $\alpha$ increases to infinity, so that $L_{1}$ is coarsely equivalent to $L$. Note that if $L$ is symmetric, then so is $L_{1}$.

4. If $L$ is a length, then $L^{\prime}(x)=L(x)+L\left(x^{-1}\right)$ is a symmetric length. We have ( $L$ bounded $) \Leftrightarrow\left(L^{\prime}\right.$ bounded) and ( $L$ proper $) \Rightarrow\left(L^{\prime}\right.$ proper $)$, but $L^{\prime}$ can be proper although $L$ is not. In particular, they are not necessarily coarsely equivalent; when it is the case, $L$ is called coarsely symmetric. For instance, the semigroup word length in $\mathbf{Z}$ with respect to the generating subset $\{n \geq-1\}$ is not coarsely symmetric.

It is well-known that a locally compact group is $\sigma$-compact (i.e. a countable union of compact subsets) if and only if it possesses a proper length. Trivially, this is a sufficient condition. Let us recall why it is necessary: let $\left(K_{n}\right)$ be a sequence 
of compact subsets covering $G$; we can suppose that $K_{1}$ has non-empty interior. Define by induction $M_{1}=K_{1}$ and $M_{n}$ as the set of products of at most 2 elements in $M_{n-1} \cup K_{n}$. Then $L(g)=\inf \left\{n \mid g \in M_{n}\right\}$ satisfies the quasi-ultrametric axiom $L(x y) \leq \max (L(x), L(y))+1$ and is symmetric and proper.

\section{Elementary results on lengths}

Lemma 3.1. Let $G$ be a locally compact group and $K$ a compact normal subgroup. Then $G$ has Property $P L$ if and only if $G / K$ has Property $P L$.

Proof. The forward implication is trivial. Conversely if $G / K$ has Property PL and $L$ is a length on $G$, then $L^{\prime}(g)=\sup _{k \in K} L(g k)$ is a length as well, so is either bounded or proper, and $L \leq L^{\prime} \leq L+\sup _{K} L$, so $L$ is also either bounded or proper.

Lemma 3.2. Suppose that $G$ has three closed subsets $K, K^{\prime}, D$ with $K, K^{\prime}$ compact, and $G=K D K^{\prime}$. Then a length on $G$ is bounded (resp. proper) if and only its restriction to $D$ is so.

Proof. Suppose that a length $L$ on $G$ is proper on $D$. Let $\left(g_{n}\right)$ in $G$ be bounded for $L$. Write $g_{n}=k_{n} d_{n} \ell_{n}$ with $\left(k_{n}, d_{n}, \ell_{n}\right) \in K \times D \times K^{\prime}$. Then $L\left(d_{n}\right)$ is bounded. As $L$ is proper on $D$ and bounded on $K$ and $K^{\prime}$, it follows that $\left(d_{n}\right)=\left(k_{n}^{-1} g_{n} \ell_{n}^{-1}\right)$ is bounded; therefore $\left(g_{n}\right)$ is bounded as well. So $L$ is proper on all of $G$. The case of boundedness is even easier.

As a consequence we get

Lemma 3.3. Let $G$ be a locally compact group and $H$ a cocompact subgroup. If $H$ has (strong) Property PL, then $G$ also has (strong) Property PL.

The converse is not true, even when $H$ is normal in $G$, in view of Proposition 1.8.

Proof of Propositions 1.8 and 1.9. Let $L$ be a semigroup length on $G$. If $L$ is not proper, then there exists an unbounded sequence $\left(a_{i}\right)$ in $\mathbf{R}^{n}$ with $L\left(a_{i}\right) \leq M$ for some $M<+\infty$ independent on $i$. Using transitivity of $K$, if $M^{\prime}=M+2 \sup _{K} L$, then for every $i$, the length $L$ is bounded by $M^{\prime}$ on the sphere $S\left(a_{i}\right)$ of radius $a_{i}$ centered at 0 . As every element of the ball $D\left(a_{i}\right)$ of radius $a_{i}$ centered at 0 is the sum of two elements of $S\left(a_{i}\right)$, it follows that $L$ is bounded by $2 M^{\prime}$ on $B\left(a_{i}\right)$. As $a_{i} \rightarrow \infty, L$ is bounded on $\mathbf{R}^{n}$, and hence $L$ is bounded on all of $G$.

Proposition 1.9 is proved in an analogous way, using the trivial fact that in any non-Archimedean local field $\mathbf{K}$, for any $m \geq n$, any element of valuation $m$ is sum of two elements of valuation $n$. 
Proof of Proposition 1.3. Define $L(g)$ as the least $n$ such that $g \in S^{n}$ and observe that $L$ is bounded on compact subsets because by the Baire category theorem, $S^{k}$ has non-empty interior for some $k$. If $S$ is symmetric, then $L$ is a length. So the assumption implies that either $L$ is proper (and hence $S$ is bounded) or $L$ is bounded (and hence $S^{n}=G$ for some $n$ ).

Conversely, suppose that $G$ is compactly generated and the condition holds. Let $L$ be a non-proper semigroup length (resp. length) on $G$. Set $S_{n}=L^{-1}([0, n])$, which is symmetric if $L$ is a length. By non-properness, there exists $n_{0}$ such that $S_{n_{0}}$ is unbounded. As $G$ is compactly generated, $S_{n}$ generates $G$ for some $n \geq n_{0}$. Then, by assumption, every element of $G$ is product of a bounded number of elements from $S=S_{n}$. By subadditivity, this implies that $L$ is bounded on $G$.

Proof of Proposition 1.2. (ii') $\Rightarrow$ (ii) is trivial. (i) $\Rightarrow$ (ii') Let $G$ act on the nonempty metric space by $C$-Lipschitz maps, and define $L(g)=d\left(x_{0}, g x_{0}\right)$ for some $x_{0}$ in $X$. Then $L$ satisfies the inequality $L(g h) \leq L(g)+C L(h)$ for all $g, h$. By the remarks at the beginning of this Section 2, $L$ is a weak length, so is coarsely equivalent to a length. So $L$ is either proper or bounded. (ii) $\Rightarrow(\mathrm{i})$ This follows from the fact that any length vanishing at 1 , is of the form $d\left(x_{0}, g x_{0}\right)$ for some isometric action of $G$ on a metric space, and 1. in Section 2.

Of course (ii) implies (iii). The converse follows from the construction in $\mathbb{N P}$, Section 5]: every metric space $X$ embeds isometrically into an affine Banach space $B(X)$, equivariantly, i.e. so that any isometric group action on $X$ extends uniquely to an action by affine isometries on $B(X)$.

\section{Lengths on semisimple groups}

Let us now proceed to the proof of Theorem 1.4.

\subsection{Lengths on the affine group}

Let $\mathbf{K}$ be a local field, and $D$ a cocompact subgroup of $\mathbf{K}^{*}$.

Proposition 4.1. Let $L$ be a symmetric length on $\mathbf{K} \rtimes D$. If $L$ is non-proper on $D$, then $L$ is bounded on $\mathbf{K}$.

Proof. Fix $W$ a compact neighborhood of 1 , so that $L$ is bounded by a constant $M$ on $W$. Suppose that the length $L$ is not proper on $D$ : there exists an unbounded sequence $\left(a_{n}\right)$ in $D$ such that $L\left(a_{n}\right)$ is bounded by a constant $M^{\prime}$. Let $u$ be any element of the subgroup $\mathbf{K}$. Replacing some of the $a_{n}$ by $a_{n}^{-1}$ if necessary, we can suppose that $a_{n} u a_{n}^{-1} \rightarrow 1$ (we use that $L$ is symmetric). Then for $n$ large enough, $w_{n}=a_{n} u a_{n}^{-1} \in W$, on which $L$ is bounded by $M$. Writing $u=a_{n}^{-1} w_{n} a_{n}$, we obtain that $L(u) \leq M+2 M^{\prime}$. 
Remark 4.2. Proposition 4.1 is false for semigroup lengths. Indeed, the subset

$$
\{(x, \lambda) \in \mathbf{K} \rtimes D:|x| \leq 1,0<|\lambda| \leq M\}
$$

generates $\mathbf{K} \rtimes D$ provided $M$ is large enough; the corresponding semigroup word length is obviously non-proper, and is easily checked to be unbounded on $\mathbf{K}$.

Remark 4.3. Proposition 4.1 still holds if the normal subgroup $\mathbf{K}$ is replaced by a finite-dimensional $\mathbf{K}$-vector space, $D$ acting by scalar multiplication.

\subsection{Case of $\mathrm{SL}_{2}$}

Denote $G=\mathrm{SL}_{2}(\mathbf{K})$, and $D, U$, and $K$ the set of diagonal, unipotent, and orthogonal matrices in $G$. Let $L$ be any semigroup length on $G$.

We have a Cartan decomposition $G=K D K$, which implies by Lemma 3.2 that boundedness and properness of the length $L$ on $G$ can be checked on $D$.

The matrix $M=\left(\begin{array}{cc}0 & -1 \\ 1 & 0\end{array}\right)$ conjugates any matrix in $D$ to its inverse. It follows that $L(g)+L\left(g^{-1}\right)$ is equivalent to $L$ on $D$, and hence on all of $G$. In other words, we can suppose that the length $L$ is symmetric.

So if $L$ is non-proper, then $L$ is bounded on $U$ by Proposition 4.1. Similarly, $L$ is bounded on $U^{t}$, the lower unipotent subgroup of $G$ (this also follows from the fact that $U^{t}$ is conjugate to $U$ by $M$ ). As every element of $G$ is product of four elements in $U \cup U^{t}$, we conclude that $L$ is bounded on $G$.

\subsection{Reduction to $G$ simply connected}

Let $H \rightarrow G$ be the (algebraic) universal covering of $G$. Then the map $H_{\mathbf{K}} \rightarrow G_{\mathbf{K}}$ has finite kernel and cocompact image. Therefore, by Lemmas 3.3 and 3.1 strong Property PL for $G_{\mathbf{K}}$ follows from strong Property PL for $H_{\mathbf{K}}$.

So we can assume $G$ algebraically simply connected, and it will be convenient and harmless to identify $G$ with $G_{\mathbf{K}}$. Let $d \geq 1$ be the K-rank of the simply connected $\mathbf{K}$-simple group $G$ and $D$ be a maximal split torus in $G$. The Cartan decomposition tells us that there exists a compact subgroup $K$ of $G$ such that $G=$ $K D K$ (in the case of Lie groups, see [He, Chap. IX 1.]; in the non-Archimedean case, see Bruhat-Tits [BrT, Section 4.4]).

So the proof consists in proving that if a semigroup length $L$ on $G$ is not proper on $D$, then it is bounded.

\subsection{Rank one}

This case is not necessary for the general case but we wish to point out that then the conclusion is straightforward. Indeed, if $G$ is such a group, then its subgroup $D$ is contained in a subgroup isomorphic to $\mathrm{SL}_{2}(\mathbf{K})$ or $\mathrm{PSL}_{2}(\mathbf{K})$ and therefore every length on $G$ is either proper or bounded on $D$. 


\subsection{General case}

Remains the case of higher rank groups. Let $W$ be the relative Weyl group of $G$ with respect to $D$, that is normalizer of $D$ (modulo its centralizer). Let $D^{\vee} \simeq \mathbf{Z}^{d}$ be the group of multiplicative characters of $D$, that is $\mathbf{K}$-defined homomorphisms from $D \simeq \mathbf{K}^{* d}$ to the multiplicative group $\mathbf{K}^{*}$.

Then by [BoT, Corollary 5.11], the relative root system is irreducible, so that by [Bk, Chap. V.3, Proposition 5(v)], the action of $W$ on $D^{\vee} \otimes_{\mathbf{z}} \mathbf{R}$ is irreducible.

If $u$ is a function $D \rightarrow \mathbf{R}_{+}$, we say that a sequence $\left(a_{n}\right)$ in $D$ is $u$-bounded if $\left(u\left(a_{n}\right)\right)$ is bounded.

Let $\Gamma \subset D^{\vee}$ be the set of $\alpha \in D^{\vee}$ such that every $L$-bounded sequence $\left(a_{n}\right)$ in $D$ is also $v \circ \alpha$-bounded, where $v(\lambda)=\log |\lambda|$ by definition. Then $\Gamma$ is a subgroup of $D^{\vee}$. It is easy to check that $D^{\vee} / \Gamma$ is torsion-free and that $\Gamma$ is $W$-invariant. On the other hand, by irreducibility, either $\Gamma=\{0\}$ or $\Gamma$ has finite index in $D^{\vee}$. As $D^{\vee} / \Gamma$ is torsion-free, this means that either $\Gamma=\{0\}$ or $\Gamma=D^{\vee}$.

Suppose that $L$ is not proper. Then there exists an sequence $\left(a_{n}\right)$ in $D$ which is $L$-bounded but not bounded. So there exists $\alpha \in D^{\vee}$ such that $v \circ \alpha\left(a_{n}\right)$ is unbounded. It follows that $\Gamma \neq D^{\vee}$. So $\Gamma=\{0\}$. In particular, for every relative root $\alpha$, there exists a sequence $\left(a_{n}\right)$ which is $L$-bounded but not $\alpha$-bounded. The argument of $\mathrm{SL}_{2}$ implies that $L$ is bounded on $U_{\alpha}$, and therefore for any root $\alpha$, $L$ is bounded on $D_{\alpha}=\left[U_{\alpha}, U_{-\alpha}\right]$. As any element of $D$ is a product of $d$ elements in $\bigcup D_{\alpha}$, we obtain that $L$ is bounded on $D$.

\section{Auxiliary results}

Proof of Lemma 1.7. By the argument of Lemma 4.3, we can suppose that $G$ is simply connected.

Let $L$ be a length on $H \times A$, and suppose that $L$ is proper on both $H$ and $A$. Suppose that $L$ is not proper. Then there exists a sequence $\left(h_{n}, a_{n}\right)$ tending to infinity in $H \times A$ so that $L\left(h_{n}, a_{n}\right)$ is bounded. As $L$ is bounded on compact subsets and is proper in restriction to the factor $A$, the sequence $\left(h_{n}\right)$ tends to infinity. By Lemma 5.1 below, there exist bounded sequences $\left(k_{n}\right),\left(k_{n}^{\prime}\right)$ and $u$ in $G(\mathbf{K})$ such that, writing $d_{n}=k_{n} h_{n} k_{n}^{\prime}$, the sequence of commutators $\left(\left[d_{n}, u\right]\right)$ is unbounded. Note that $L\left(d_{n}, a_{n}\right)$ is bounded as well.

Suppose that $L\left(d_{n}^{-1}, a_{n}^{-1}\right)$ is bounded (this holds if $L$ is assumed coarsely symmetric). Now $L\left(\left[\left(d_{n}, a_{n}\right),(u, 1)\right]\right)=L\left(\left[d_{n}, u\right], 1\right)$ is bounded. But this contradicts properness of the restriction of $L$ to $H$.

If $L\left(d_{n}^{-1}, a_{n}^{-1}\right)$ is not assumed bounded, we can go on as follows. First note that the proof of Lemma 5.1 provides $\left(d_{n}\right)$ as a sequence in the maximal split torus $D$, and we assume this. If $W$ denotes the Weyl group of $D$ in $H$, then for every $d \in D$ the element $\prod_{w \in W} w d w^{-1}$ of $D$ is fixed by $W$, so is trivial. 
Now the sequence

$$
L\left(\prod_{w \in W}(w, 1)\left(d_{n}, a_{n}\right)\left(w^{-1}, 1\right)\right)=L\left(1, a_{n}^{|W|}\right)
$$

is bounded. Therefore, by properness on $\{1\} \times A$, the sequence $\kappa_{n}=a_{n}^{|W|}$ is bounded. Thus, the sequence $L\left(1, \kappa_{n}^{-1}\right)$ is bounded. Now the sequence

$$
L\left(\prod_{w \in W-\{1\}}(w, 1)\left(d_{n}, a_{n}\right)\left(w^{-1}, 1\right)\right)=L\left(\left(d_{n}^{-1}, a_{n}^{-1}\right)\left(1, \kappa_{n}\right)\right)
$$

is bounded in turn, so $L\left(d_{n}^{-1}, a_{n}^{-1}\right)$ is bounded, and this case is settled.

Lemma 5.1. Let $\mathbf{K}$ be a local field and $G$ a simple simply connected linear algebraic group over $\mathbf{K}$. Let $\left(g_{n}\right)$ be an unbounded sequence in $G(\mathbf{K})$. Then there exist bounded sequences $\left(k_{n}\right),\left(k_{n}^{\prime}\right)$ and $u$ in $G(\mathbf{K})$ such that the sequence of commutators $\left(\left[k_{n} g_{n} k_{n}^{\prime}, u\right]\right)$ is unbounded.

Proof. By the Cartan decomposition (see Paragraph 4.3), we first pick $\left(k_{n}\right)$ and $\left(k_{n}^{\prime}\right)$ such that $a_{n}=k_{n} g_{n} k_{n}^{\prime}$ belongs to $D$, the maximal split torus. There exists one weight $\alpha$ such that $\alpha\left(a_{n}\right)$ is unbounded. Fix $u$ in the unipotent subgroup $G_{\alpha}$. Then $a_{n} u a_{n}^{-1}$ is unbounded, so $\left[a_{n}, u\right]$ is unbounded as well.

Proposition 1.6 follows from Lemma 1.7 when $G$ is a direct product of simple groups. The general case follows by passing to the (algebraic) universal covering $\tilde{G}$ of $G$, as $\tilde{G}(\mathbf{K})$ maps to $G(\mathbf{K})$ with finite kernel and cocompact image.

\section{References}

[Be] G. Bergman. Generating infinite symmetric groups. Bull. London Math. Soc. 38 429-440, 2006.

[Bk] N. Bourbaki. Éléments de Mathématique. Groupes et algèbres de Lie. Chap 4-6. Hermann, 1968.

[BoT] A. Borel, J. Tits. Groupes réductifs. Publ. Math. Inst. Hautes Études Sci. 27, 55-151, 1965.

[BrT] F. Bruhat, J. Tits. Groupes réductifs sur un corps local. I, Données radicielles valuées. Publ. Math. Inst. Hautes Études Sci. 41, 5-251, 1972.

[C] Y. de Cornulier, Strongly bounded groups and infinite powers of finite groups. Comm. Algebra 34, 2337-2345, 2006. 
[CTV] Y. de Cornulier, R. Tessera, A. Valette. Isometric group actions on Banach spaces and representations vanishing at infinity. Transform. Groups 13, no. 1, 125-147, 2008.

[He] S. Helgason. Differential Geometry, Lie Groups, and Symmetric Spaces. Academic Press, New York 1978.

[Hj] G. Hjorth. Classification and orbit equivalence relations. Mathematical Surveys and Monographs, 75. American Mathematical Society, Providence, RI, 2000.

[NP] L. Nguyen Van Thé, V. Pestov. Fixed point-free isometric actions of topological groups on Banach spaces. Bull. Belg. Math. Soc. Simon Stevin 17 (2010), no. 1, 29-51.

[Sh] Y. Shalom. Rigidity, unitary representations of semisimple groups, and fundamental groups of manifolds with rank one transformation group. Annals of Math. 152 113-182, 2000.

[St] R. Struble. Metrics in locally compact groups. Compos. Math. 28(3), 217-222, 1974. 